\title{
Consideraciones para el Código de ética empresarial de las empresas afiliadas a la AMAV-México
}

\section{Considerations regarding the code of business ethics for companies affiliated with AMAV-Mexico}

\section{Considerações para o código de Ética empresarial das empresas filiadas com a AMAV-México}

\author{
Manuel A. Garzón-Castrillón ${ }^{1}$; Luis A. Pérez-Romero ${ }^{2}$ \\ 1 BA, MSc, PhD, Post PhD, Profesor Doctorado en Gestión Universidad EAN \\ 2 MD. MSc. PhD, Profesor e investigador del ITESM (Instituto Tecnológico y Estudios Superiores de Monterrey) \\ Email: manuelalfonsogarzon@fidee.org
}

Recibido: diciembre 23 de $2015 \quad$ Aceptado: junio 27 de 2016

\begin{abstract}
Resumen
Esta publicación tiene como objetivo proponer unas consideraciones para el código de ética empresarial, con base en el grado de importancia que tiene para las empresas afiliadas a la AMAV-México, orientado a integrar el enfoque ético sostenible, con el modelo económico empresarial al medio ambiente y al desarrollo social en los procesos de negocios y proporcionar herramientas necesarias para que se implemente este enfoque sostenible en las pequeñas y medianas empresa, teniendo en cuenta que los códigos de ética en las pymes pueden constituirse en un medio eficaz para institucionalizar la ética dentro de las organizaciones, si se presentan como la expresión clara y definida de los valores compartidos a su interior, este artículo pretende mostrar que su utilidad está estrechamente vinculada al desarrollo de virtudes de las personas involucradas, a una adecuada política de comunicación y a un firme liderazgo, comprometido con los valores morales que refleje la conducta moral sostenible de quienes la conforman.
\end{abstract}

Palabras clave: ética, ética empresarial, código de ética empresarial

\begin{abstract}
This article advances some considerations regarding a code of business ethics, based on the degree of importance apportioned by companies affiliated to AMAV-Mexico, orientated towards integrating an ethical approach which is sustainable with a business economic model regarding the environment and social development concerning business matters. It would thus provide the necessary tools for implementing such sustainable approach in small- and medium-sized companies (SMSC). It should be born in mind that SMSC codes of ethics can become an effective means of institutionalising ethics within organisations if they are presented as a clear and defined expression of their shared values. This article is aimed at showing that their usefulness is closely linked to the development of the merits of the people involved, a suitable communication policy and firm leadership, committed to the moral values reflecting the sustainable moral conduct of those who work in SMSC.
\end{abstract}

Key words: ethics, business ethics, code of business ethics. 


\section{Resumo}

Esta publicação tem como objetivo propor algumas considerações para o código de ética de negócios, com base no grau de importância para as empresas afiliadas com a AMAV-México, visando a integração de abordagem ética sustentável do negócio modelo econômico do ambiente e desenvolvimento social nos processos de negócios e fornece ferramentas necessárias para fazer esta abordagem sustentável é implementado em pequenas e médias empresas, considerando que os códigos de ética nas PME podem tornar-se um meio eficaz para institucionalizar a ética dentro organizações, se apresenta como uma expressão clara e definida de compartilhados seus valores internos, este artigo tem como objetivo mostrar que a sua utilidade está intimamente ligada ao desenvolvimento das virtudes das pessoas envolvidas, para uma política adequada de comunicação e liderança forte, comprometida com os valores morais que refletem a conduta moral sustentável daqueles que a conformam.

Palavras-chave: ética, ética empresarial Código de Ética Empresarial

\section{Introducción}

El tema de la ética afecta al mundo de las organizaciones, y tiene como objetivo principal valorar su capacidad de ser compatible con la creación del valor, este dilema se genera porque el aumento de la competitividad impone el desarrollo de obligaciones de transparencia por encima de los niveles establecidos en la normativa, lo cual implica transparentar los procesos para el escrutinio ético de las operaciones de las empresas, y que en las pymes no ha sido estudiado.

En este aspecto relacionado con la ética empresarial en las pymes encontramos que se presenta escasez de conocimientos por parte de los directivos de las Pequeñas y Medianas Empresas (pymes) como lo muestra Larrán et al., (2011), se presenta poca cercanía de este tipo de organizaciones con sus stakeholders Spence y Rutherfoord, (2003); Worthington et al., (2006); Perrini, (2006); Fisher et al., (2009); Russo y Tencati, (2009) de la mima forma se caracterizan estas organizaciones por la no utilización de mecanismos formales de comunicación Spence y Lozano, (2000); Graafland et al., (2003); Nielsen y Thomsen, (2009), y todos estos aspectos han sido señalados como posibles razones del estado incipiente de la investigación desarrollada hasta el momento según Vázquez-Carrasco y LópezPérez, (2012) sobre la ética empresarial en las PYMES.

En este orden de ideas Herrera y Abreu (2008) proponen un enfoque metodológico de planeación, ejecución, verificación y evaluación para incorporar prácticas socialmente responsables en las Pequeñas y Medianas Empresas, las cuales se han incorporado al proceso de rendir cuentas más allá de los resultados económicos y financieros, reflejados en informes de responsabilidad social denominados reportes de sostenibilidad y que con base en la teoría de empresa que manejen los directivos, en cada organización se abordará de forma diferente, en razón a que el desarrollo del concepto de Responsabilidad Social es básicamente un proceso de reflexión ética.
Esta publicación tiene como objetivo proporcionar unas consideraciones para el código de ética empresarial de las Pymes afiliadas a la AMAV-México, dada la importancia que tiene el integrar el modelo económico empresarial al medio ambiente y al desarrollo social en los procesos de negocios y proporcionar herramientas necesarias para que se implemente este enfoque sostenible en estas pequeñas y medianas empresa.

Proponer unas consideraciones para el Código de ética empresarial, con base en el grado de importancia que tiene para las empresas afiliadas a la AMAV-México, integrar el enfoque ético sostenible.

Con base en el estudio realizado se logró construir un marco de referencia para proponer unas consideraciones que les permitan integrar el código de ética sostenible que contribuya a la implementación de la Responsabilidad Social empresarial integrada en la ISO 26000 para las Pymes (Agencias de Viajes) afiliadas a la AMAV-México.

\section{Marco de referencia}

\section{Ética}

Para poder abordar el tema de la ética empresarial, empezaremos por establecer etimológicamente que "ética" -del griego ethos, carácter- alude más a la raíz del problema, pues el comportamiento es la cuna del carácter, de lo que uno hace con su vida. El ethos alude al comportamiento como la forma de forjar el carácter. Así pues, para Román (2000) la ética estudia el fenómeno humano de las morales con el objetivo moral de que éstas lo sean auténticamente: es decir, que orienten a los humanos en la tarea vital de vivir bien; que los orienten en la labor de vivir conforme ellos libremente decidan, lo que supone unas morales que, más allá del interés dogmático, permitan la generación de seres humanos auténticos, con carácter, por tanto la ética versa sobre lo que debe ser, trata sobre 
lo que puede ser de diferentes formas, sobre la acción correcta.

De lo dicho hasta aquí se desprende siguiendo a GuiIlen (2010) que la ética es una ciencia teórica, a la vez que un tratado práctico, ya que se refiere a las acciones humanas no sólo para conocerlas sino principalmente para dirigirlas. Por otra parte, la ética considera las acciones humanas en su relación con el modo de ser (ethos) que la persona adquiere a través de ellas.

\section{Ética empresarial}

Las definiciones que existen sobre la Ética Empresarial son muy diversas, así Suarez (2011) ; Martin (2000) y MacDonald, (2010) coinciden en que los temas son directamente los de la ética personal y organizacional, como los códigos de buena conducta de alcance integral para todos los que laboran en la empresa, autorregulación de las organizaciones y los códigos de buena conducta para los gobiernos corporativos, es decir, para representantes de accionistas -directores-y gerentes de primera línea en la estructura organizacional, no es un ente abstracto, habla de los valores del hombre de negocios en este momento, y de la jerarquización que hace de sus valores frente a los dilemas de la gestión, que complementa por Wigodski, (2009) que debe ser una característica que se adquiere en la formación profesional de los actores del mundo de los negocios, de manera que en los procesos de toma de decisiones los empresarios velen por el respeto de la dignidad de los grupos de interés, como una garantía para la supervivencia y competitividad de sus organizaciones a largo plazo.

De esta manera corresponde con la propuesta de Melé, (1991) en relación con la ética empresarial, al plantear los múltiples problemas que implica un comportamiento honrado en los negocios; ya que es misión de la ética en general, indicar los principios básicos que orientan una correcta conducta humana donde quiera que se dé. Este será precisamente el papel de la ética empresarial, cuyo objeto consiste en aplicar los principios éticos generales a las características peculiares de la empresa y de los negocios.

Lo importante en relación con la ética es que los autores más representativos coinciden en que la ética genera buenos negocios y Argandoña (1990) complementa que: "la falta de ética es siempre rentable para algunos, a corto plazo. Y, sin embargo, la ética es siempre rentable para el conjunto de la sociedad, a largo plazo".
Por tanto, siguiendo a Cano (2011) los autores más representativos que han escrito sobre ética empresarial muestran las dos direcciones de la reflexión ética, por un lado, la ética centrada en la conducta y la perspectiva ética como un proceso reflexivo, de la ética empresarial, ambas perspectivas no son mutuamente excluyentes, incluso se complementan, la diferencia sería en todo caso decidir cuál aplicar en cada situación.

\section{Antecedentes de la Ética empresarial}

El interés por la ética empresarial se produce en Estados Unidos comenzada la década de los setenta del siglo pasado. Gómez Pérez (1990), establece que, en los países socialista, Japón o Europa, se presenta por la falta de libertad de empresa en el caso de los primeros; y la existencia de principios éticos profundamente arraigados en la cultura milenaria japonesa para los segundos.

Revisando la época en la que en Estados Unidos empieza a preocuparse por la ética empresarial, ésta coincide cronológicamente para Guillen (2010) con la oleada de escándalos ocurrida en la segunda mitad de la década de los setenta: el asunto Watergate, que ocasionó la caída del presidente Nixon en 1974, el descubrimiento de sobornos que implicaban a grandes compañías, unos años antes, la aparición de nuevos problemas en otras disciplinas como la medicina o las leyes, originaron una especial sensibilidad por las cuestiones éticas, que también se han visto trasladadas al mundo empresarial.

El hecho de que sólo a partir de los años 70 se comenzara a hablar en Estados Unidos de ética empresarial para Román (2000) no debe inducir a pensar que, porque no existiera el nombre, tampoco existía una idea de cuál había de ser el comportamiento que debía seguirse en los negocios. Predominaba una determinada moral bastante identificada con la religión, y existía una moral de la empresa, la moral del dueño, sin que nadie pidiera más justificaciones. No se trata de que, como algunos suponen, el discurso moral sea ajeno al discurso propiamente empresarial, de que las reglas del juego de la economía y las de la empresa sean ganar dinero -esto es, hacer negocio- y de que hacerlo bien signifique, como mucho, estar dentro de la ley.

Lo anterior teniendo en cuenta que durante mucho tiempo según Echaniz (2003) el mundo de la empresa se ha regido bajo la máxima de «los negocios son los negocios». Esta afirmación, llevada al extremo, conduce a un «todo vale para conseguir beneficios» que es difícilmente defendible y, desde luego, nada deseable socialmente. La conclusión es clara: la formación de 
buenos profesionales (con los conocimientos técnicos necesarios) no necesariamente conduce a la formación de profesionales «buenos».

La historia de la ética empresarial en Estados Unidos puede considerarse según De George (2011) como la intersección entre tres corrientes entrelazadas. Cada una de estas puede dividirse a su vez en al menos dos ramas correspondientes. La primera corriente, a la que De George denomina corriente de la ética en los negocios, se refiere a la larga tradición de aplicar a los negocios las normas de la ética, del mismo modo que se han aplicado a otros aspectos de la vida social y personal.

La segunda corriente para De George (2011) corresponde al desarrollo de una disciplina académica que ha recibido el nombre de ética empresarial. Esta se divide asimismo en dos ramas principales, siendo una la rama de la ética empresarial filosófica, normativa y crítica, y la otra la rama científica y social, principalmente descriptiva y empírica.

La tercera corriente siguiendo a De George (2011) está relacionada con la adopción en los negocios de la ética o, al menos, de lo que esta implica, que se subdivide en la integración de la ética en los negocios y la práctica empresarial, por un lado, y el compromiso de responsabilidad social de la empresa por otro.

En el anexo 1, se relacionan los eventos más destacados que se realizaron entre 1976 y 2012. Así mismo es importante considerar que en la década de los noventa en los estados unidos de norte américa, se asiste al nacimiento e implementación en las empresas de códigos internos de reglas de conducta. Álvarez Rivas y De la Torre (2002) indican que, en 1987, el 75 por 100 de las mayores 300 empresas norteamericanas habían establecido tales códigos.

\section{Códigos de ética empresarial como instrumentos para la comunicación de la cultura organizacional}

De forma creciente en el siglo XXI se han venido transmitiendo los distintos aspectos de la cultura organizacional a través de instrumentos escritos: enunciados de misión y valores, credos, políticas corporativas y códigos formales de ética. Este último instrumento es el que muestra el crecimiento más significativo: para Lozano (2001) si en 1984-1985 el 75\% de las 1000 mayores empresas del mundo tenía un código de ética, ese porcentaje ya había alcanzado el $93 \%$ en 1989 1990. Y ha seguido creciendo, ante lo cual surge la pregunta, a la luz de los conocidos escándalos, ¿cuán eficaces han sido esos códigos o si su implementación tan sólo ha respondido a una moda o a una demanda de la sociedad a la cual se responde como parte de una retórica corporativa tendiente a crear un capital de reputación?

Para Adela Cortina (1996), es la propia actividad de la organización la que exige un tipo de valores, principios, actitudes, formas determinadas de comportamiento, que obligan a quienes se incorporan a ellas, a que las plasmen o no, estos y estas actitudes y valores en códigos de conducta. Por tanto, no sólo los individuos son moralmente responsables, sino también las organizaciones.

De esta manera para Montuschi (2010) los códigos de ética, en particular, deberían dar respuesta a los planteamientos y dilemas morales que se presentan en las organizaciones y que, en ciertas ocasiones, pueden asumir dimensiones inusuales de dramatismo. En general, se supondría que estos códigos articulan los valores de la organización con las responsabilidades, obligaciones y objetivos éticos de la misma y de sus integrantes.

Unos códigos de ética afirman Giddens (1979; Nascimento (2000); Schwartz, (2005), y Gonzalez et al., (2006) pueden ser entendidos como o conjunto de reglas de conducta que deben ser observadas por los miembros de una profesión o, de un sector de actividad o de una empresa. Las reglas y los principios de un código de ética empresarial establecen el encuadramiento normativo da empresa, o sea, los criterios de orientación para decidir o que es correcto y que no lo es, pretendiendo así asegurar el comportamiento ético de sus miembros y en el mismo orden de ideas.

Si bien para Deck (2002) se los suele englobar bajo la denominación genérica de "códigos de ética" es importante diferenciar claramente tres tipos de instrumentos o códigos cuyo propósito difiere:

- Códigos de ética (propiamente dichos): Para Deck (2002) contienen enunciados de valores y principios referidos al propósito de la organización, a sus obligaciones y responsabilidades hacia los stakeholders y a las normas que deberán conformar el comportamiento de sus integrantes. También se los suele identificar como "códigos basados en valores".

- Códigos de prácticas: Para Deck (2002) deben enumerar, interpretar e ilustrar las normas que deben guiar la toma de decisiones en diferentes instancias y las acciones concretas de los integrantes de la corporación que conformen los principios y valores enunciados en el punto 1. 
- Códigos de conducta: Para Deck (2002) constituyen enunciados de reglas expresadas de forma afirmativa o negativa acerca de aquello que pueden o no pueden hacer los integrantes de la organización, pueden fijar penalidades por incumplimiento y fijar los procedimientos para hacer cumplir las normas y apelar en caso de disenso o conflicto de intereses.

La eficacia de un código de ética según Debeljuh (2005) se relaciona estrechamente con el grado de compromiso de las personas con los valores que él contiene, de esta manera refuerza Debeljuh (2005), afirma que el verdadero cometido de un código de ética ha de ser ayudar a la excelencia en el trabajo de todas las personas y, en concreto, facilitarles el desarrollo de sus virtudes y el despliegue de todas sus potencialidades.

En este aspecto es importante tener en cuenta lo que Ramos (2013) concluyó en su estudio, que la ética no puede ser impuesta por la alta dirección, debe ser auto-construida, co-construida y hetero-construida por los grupos de interés. Pero como lo plantea Herrera (2010), este tipo de documentos siguen siendo iniciativas unilaterales que no reflejan la realidad de las necesidades, expectativas y demandas que tienen los grupos de interés y que "obedecen a una serie de intereses empresariales, particularmente relacionados con la imagen ante los consumidores (afiliados) y con las exigencias de los mercados.

\section{Grupos de interés}

En primer lugar, vamos a abordar, en relación con la teoría de los grupos de interés, que la expresión proviene del neologismo inglés "stakeholder' $s$ ", traducido como grupos de interés, que nos invita a comprender que, en las organizaciones, no solo existe responsabilidad hacia los inversionistas, sino también hacia otros grupos que están interesados en su funcionamiento.

Freeman y Reed (1983) Propusieron dos definiciones de stakeholder: una acepción amplia, que incluye grupos que son amistosos u hostiles, y una acepción restringida, que captura la esencia de la definición del SRI (Stanford Research Institute), pero es más específica y una acepción amplia: cualquier grupo o individuo identificable que pueda afectar el logro de los objetivos de una organización o que es afectado por el logro de los objetivos de una organización (grupos de interés público, grupos de protesta, agencias gubernamentales, asociaciones de comercio, competidores, sindicatos, así como segmentos de clientes, accionistas y otros) y una acepción restringida: cualquier grupo o individuo identificable respecto del cual la organización es dependiente para su supervivencia (empleados, segmentos de clientes, ciertos proveedores, agencias gubernamentales clave, accionistas, ciertas instituciones financieras, y otros).

En este orden de ideas, para Freeman, (1984), la teoría de los grupos de interés, insta a mostrar que la capacidad de una empresa para generar una riqueza sostenible a lo largo del tiempo y, con ello, su valor a largo plazo viene determinada por sus relaciones con sus grupos de interés.

La propuesta de Gil, et al., (2011) se constituye en diferentes categorías con el objeto de priorizar los grupos de interés en:

- Críticos: son aquéllos que tienen un impacto clave económico, fortalecen o influyen en la reputación, conceden o limitan licencias o accesos o crean el futuro del sector.

- Básicos: son aquellos con un impacto medio en los resultados del negocio, que pueden afectar parcialmente la reputación, pero que inciden de alguna manera en los procesos clave de la compañía.

- Complementarios: son aquéllos que tienen un mínimo impacto económico, influyen escasamente en la reputación y pueden proveer de servicios o productos complementarios.

Se infiere por tanto que uno de los puntos más importantes en el ámbito de la sostenibilidad es logar el diálogo con los grupos de interés, de esta manera y teniendo en cuenta que la teoría de los grupos de interés, de Freeman (1984) Mohn (2005), quienes proponen que la capacidad de una organización para generar riqueza sostenible a lo largo del tiempo y a largo plazo, está determinada por sus relaciones con sus grupos de interés, cuya cooperación puede tener un gran éxito.

De esta manera la teoría de los grupos de interés intenta responder a las interrogantes relacionadas con los intereses respecto de los cuales las organizaciones son gestionadas Falçao y Fontes, (1999), desde dos grandes perspectivas:

- Descriptiva: Para Falçao y Fontes, (1999) ¿Qué intereses son considerados y cuáles desestimados en el actual sistema de gestión de la organización, señalando que los intereses que prevalecen son los de los accionistas por sobre el resto de los stakeholders'.

- Prescriptiva: para Falçao y Fontes, (1999) ¿Cuáles son los intereses qué deben ser privilegiados o no 
marginados para la sustentabilidad de la organización en el largo plazo, y no afectar la supervivencia organizacional?

Por tanto, la nueva mirada de la organización desarrollada por Post et al., (2002), se fundamenta en la creencia de que la capacidad de una organización para crear riqueza de manera sostenible en el tiempo, se encuentra determinada por las relaciones que se desarrollan con los diferentes grupos de interés críticos.

Finalmente, en lo relacionado con los stakeholders, los aportes de Freeman (1984) reconocidos como la primera sistematización teórica en 1984 concentra una elevada unanimidad entre los investigadores de esta temática.

\section{Metodología}

Se realizó una investigación exploratoria y descriptiva con el apoyo del método de investigación hipotético inductivo y cuantitativo (se aplicó un cuestionario estructurado) con el fundamento de modelos estadístico multivariable y la revisión del marco conceptual, teórico y de antecedentes que respalden las consideraciones para el Código de ética empresarial para las empresas afiliadas a la AMAV-México.

\section{Planteamiento del problema}

¿Cuáles variables propias de las Agencias de Viajes Afiliadas a la Asociación Mexicana de Agencias de Viajes (AMAV-México), influyen en la intención de tener un enfoque ético y sostenible de los negocios según los factores clave de un código de ética empresarial sostenible?

Unidad de análisis: Directivos y/o dueños de las Agencias de Viajes afiliadas a la AMAV-México.

Lugar: Ciudad de Aguascalientes lugar de encuentro de la Convención Nacional de los afiliados a la AMAV.

Variable dependiente: grado de importancia para implementar un enfoque ético y sostenible en sus negocios, la cual se medirá en una escala likert de 1 a 5 , siendo cinco la máxima importancia y el grado de conocimiento que se tiene sobre la ética empresarial, así como la prioridad que existe en estos momentos para implementar un código de ética empresarial, medida en escala Likert.

Variables independientes: propias de las empresas y propias de los fundadores de las mismas como: forma jurídica, antigüedad de constituida, giro, número de empleados, si tiene certificaciones en calidad u otras, datos de los presidentes o directivos de las Agencias como educación, ocupación, edad, género, grado de conoci- miento e intención de incorporar en la AMAV un código de conducta y acciones concretas para la RSE.

\section{Objetivo}

Proponer unas consideraciones para el Código de ética empresarial, con base en el grado de importancia que tiene para las empresas afiliadas a la AMAV-Méxi$\mathrm{co}$, integrar el enfoque ético sostenible.

\section{Prueba piloto del instrumento}

Al cuestionario estructurado, se le aplicó una prueba piloto con cinco directivos de las empresas afiliadas a la AMAV-México, quienes ayudaron mucho en la redacción de algunas preguntas que para la mayoría de los empresarios no comprendían. El grado de validez y confiabilidad global se obtuvo con el Alfa de Cronbrach de .75 para el cuestionario en general y se realizó la validez concurrente con bloques de variables que miden el nivel de importancia para implementar la ética empresarial con un Alfa de Cronbach de 0.84 y de 0.85 para el bloque de preguntas del cuestionario que miden solamente las actividades de la Responsabilidad Social Empresarial.

\section{Validez y confiabilidad del instrumento de medición}

Se encontró un grado de validez y confiabilidad global del cuestionario con el apoyo del Alfa de Cronbach del 0.86; un Alfa de Cronbach parcial de 0.82 para el bloque de preguntas hacia una práctica empresarial con ética; 0.79 para el bloque de preguntas relacionadas a la RSE; .883 para el bloque de preguntas sobre cómo realizarían actividades de RSE y 0.91 de posibles acciones a realizar por parte de las empresas de la Asociación Mexicana de Agencias de Viajes (AMAV). Para el bloque de los stakeholder se obtuvo un Alfa de Cronbach de 0.78. Con estos valores de la validez y confiabilidad del cuestionario y con los grados de confianza del tamaño de la muestra los datos nos ofrecen la certidumbre suficiente para realizar las estadísticas inferenciales de factor análisis y análisis discriminante.

\section{Tamaño y selección de la muestra}

Se utilizó la fórmula para el tamaño de la muestra de población finita ya que el total de los afiliados regulares es de 636 y con un error del $5 \%$ y nivel de confianza del $95 \%$ se obtuvo el siguiente tamaño de la muestra:

$$
\begin{aligned}
& \mathrm{n}=\mathrm{P}(1-\mathrm{P}) /\left(\mathrm{E}^{2} / \mathrm{Z}^{2}\right)+\mathrm{P}(1-\mathrm{P}) / \mathrm{N} \\
& \mathrm{n}=.5(1-.5) / .(05)^{2} /(1.96)^{2}+(.5)(1-.5) / 636 . \\
& \mathrm{n}=239
\end{aligned}
$$


La selección se realizó de manera aleatoria simple, la cual se fue modificando en función de la disponibilidad para contestar el cuestionario, pero procurando respetar la aleatorización.

\section{Trabajo de campo}

El trabajo de campo se realizó aplicando el cuestionario de manera directa y personalizada abordando a cada uno de los afiliados de la AMAV que quisiera colaborar de manera voluntaria a contestar el cuestionario, pero respetando la aleatorización sistematizada simple: uno de cada tres podría contestar el cuestionario.

\section{Resultados}

Con el apoyo del paquete estadístico del SPSS, se realizaron los análisis estadísticos univariados, bivariados y multivariados para dar respuesta a cada una de las tres hipótesis planteadas. Para fines prácticos del análisis se presentarán en tres grandes apartados: estadística descriptiva, estadísticos para el Código de Ética sostenible, y el análisis de las hipótesis planteadas para la ética, los cuales se describen a continuación:

Preguntas: ¿Por qué se requiere un código de ética y quiénes deben participar en el marco de referencia para construir el Código de Ética de la AMAV?

Ante estas preguntas los empresarios de la AMAV consideran que es importante un Código de Ética para Mejorar los procesos internos de las Agencias de Viajes, obtener más orden y control de los procesos, generar confianza, trabajar con más calidad y de manera honesta para poder publicar o registrar las buenas prácticas de empresas responsables y éticas. Estos seis atributos mencionados representante el $80 \%$ de las menciones realizadas y se consideran estos como un marco de referencia fundamental para integrar el Código de Ética. Figura 1.

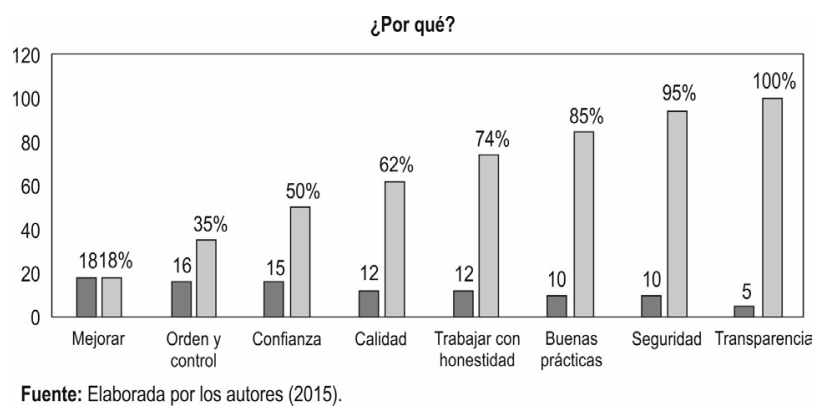

Figura 1. Atributos para integrar el Código de Ética
Pregunta: ¿Quiénes deben participar en el marco de referencia para construir el código de ética de la AMAV?

Los afiliados a la AMAV consideran importante que participen en la integración del Código de Ética todos los miembros afiliados a la AMAV y de preferencia los Presidentes o dueños de las Agencias de Viajes, siendo estos dos rubros el $80 \%$ por ciento de las menciones, por lo que se sugiere integrar a todos los afiliados de manera presencial o virtual para que participen en el diseño e integración del Código de Ética Empresarial AMAV. Ver figura 2.

Los factores internos de cada Agencia de Viajes que se deben considerar como marco de referencia para para construir el marco de referencia para el Código de Ética-AMAV son: actuar con profesionalismo, actuar con la verdad, de manera responsable, mejorando el servicio día a día e involucrar todas las áreas funcionales de la agencia de viajes como los empleados de ventas, servicio al cliente, contabilidad, administración y planeación. Tomando en cuenta estos cinco factores internos, se tendría el $90 \%$ de impacto en todos los afiliados a la AMAV. Ver figura 3.

Pregunta: ¿Qué factores internos a cada agencia de viajes, se debe integrar para construir el marco de referencia para el código de ética de la AMAV?

Los elementos en concreto que se deben tomar en cuenta para integrar el Código de Ética en la AMAV son en orden de importancia: primero la honestidad, seguida de respeto, transparencia, calidad del servicio, generar lazos de amistad, comunicación entre todos los afiliados y crear espacios de confianza. Con estos atributos se logrará tener un impacto en el 80\% de las referencias o elementos concretos sugeridos por los afiliados para integrar un Código de Ética Empresarial. Ver figura 4.

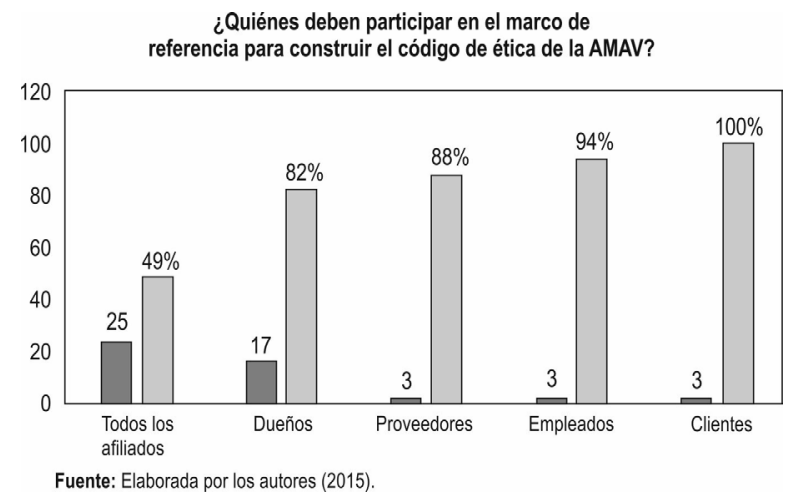

Figura 2. Participantes para diseño e integración del Código de Ética Empresarial 


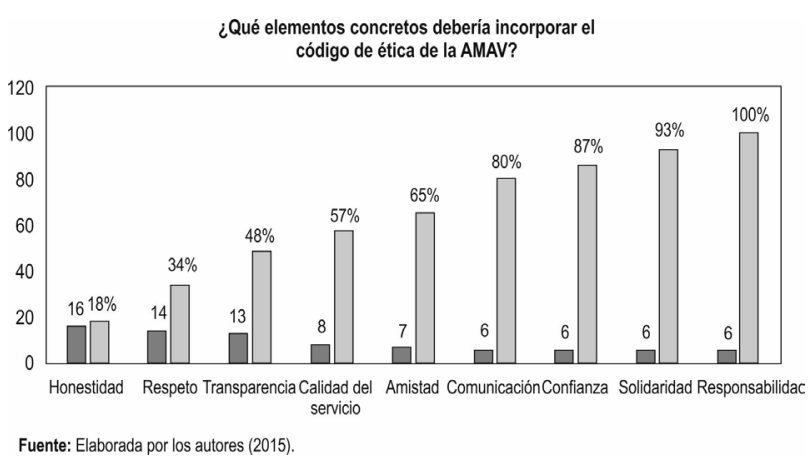

Figura 3. Factores internos para construir el marco de referencia para el código de ética

\section{Pregunta: ¿Qué elementos concretos debería incor- porar el código de ética de la AMAV?}

Las empresas de afiliadas a la AMAV-México, sugieren que se debe tener un comportamiento ético en primera instancia ante los socios, seguido de los clientes, proveedores y empleados. Con estos cuatros grupos se tendría un impacto del $90 \%$ de los grupos de interés que deben ser tomados en cuenta en la integración de un Código de Ética Empresarial AMAV-México. Lo curioso es que la mayoría quiere internalizar la idea de crear un código de ética para ellos y no para la Presidencia de la AMAV. Ver figura 5.

\section{Pregunta: ¿Ante quién deben tener un comporta- miento ético como empresa afiliada a la AMAV?}

Los factores de negociación que se deben integrar entre las agencias de viajes dentro de un marco de referencia para el Código de Ética de la AMAV y generara empoderamiento del mismo código son en orden de importancia: el respecto entre todos los colaboradores de la AMAV-México, saber trabajar en equipo en beneficio de nuestros clientes-turistas, siempre procurar trabajar con honestidad, transparencia y solidaridad sin descuidar la comunicación clara ante toda circunstancia del negocio mismo. Se sugiere integrar todos

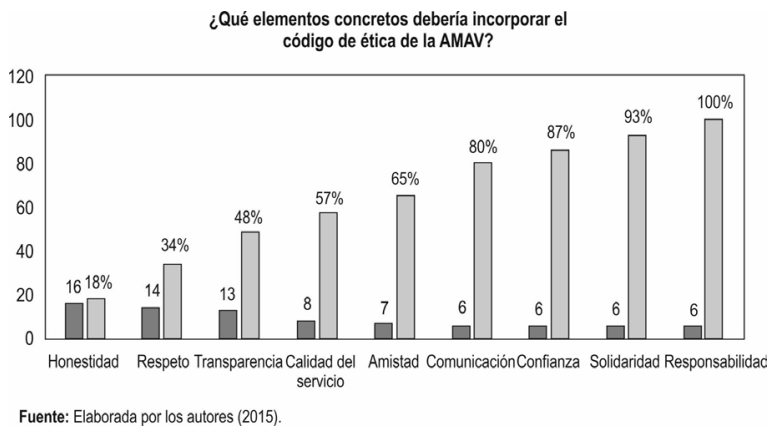

Figura 4. Atributos concretos debería incorporar el código de ética

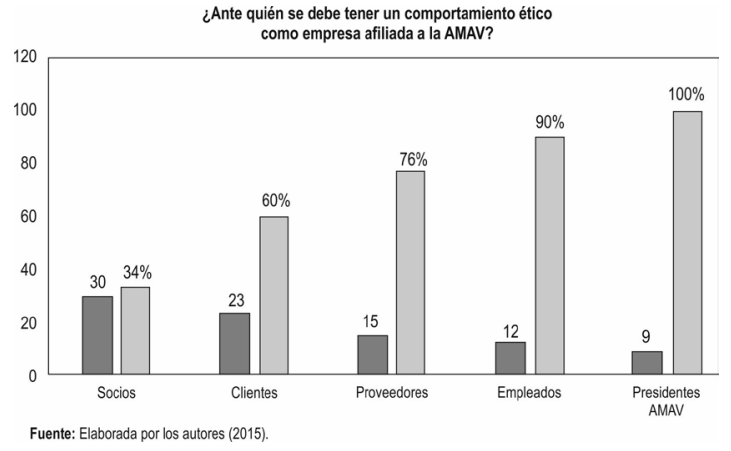

Figura 5. Comportamiento ético como empresa afiliada

estos factores para que cada uno de los afiliados realmente se sienta escuchados en esta experiencia de integrar un Código de Ética Empresarial AMAV-México. Ver figura 6.

Pregunta: ¿Qué factores de la negociación entre agencias de viajes, se deben integrar en un código de conducta ética para la AMAV?

En cuanto al grado de importancia para integrar un Código de Ética Empresarial en las Agencias incorporadas a la AMAV, la evaluación proporcionada fue de 4.9 de una evaluación máxima de 5, lo que quiere decir que el grado de importancia para integrar este código es muy alto y se debe incorporar los principios fundamentales del respeto con una evaluación de 4.9, siendo la máxima 5, la verdad con una evaluación de 4.7, la transparencia con una evaluación de 4.7, la justicia con 4.6, honestidad igual con 4.6, la solidaridad con 4.6 y la corresponsabilidad con una evaluación de 4.4. Ver tabla1.

Al agrupar los temas de la importancia de los principios fundamentales para evolucionar hacia una práctica empresarial con Ética en la AMAV-México y con el apoyo de análisis multivariable de factores, se obtuvo una $\mathrm{KMO}$ de .612, lo que indica una adecuada rota-

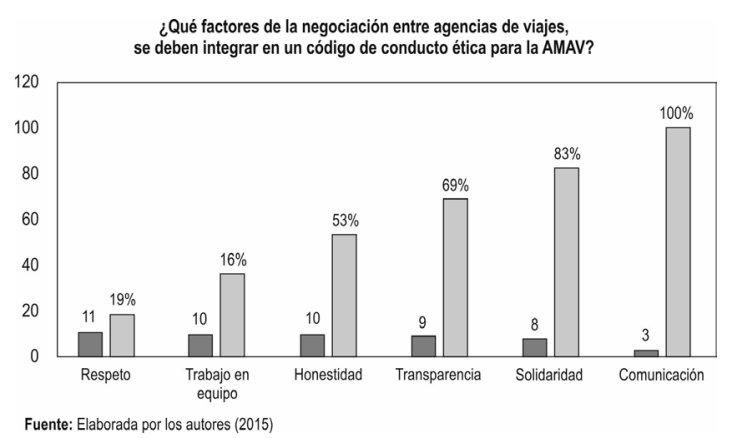

Figura 6. Factores de la negociación entre agencias 
ción de factores con una varianza explicada del $69 \%$ para los dos factores claramente definidos ante estos principios para integrar el Código de Ética Empresarial. El peso relativo de cada variable según los datos arrojados por la matriz de componentes rotados de varimax, muestra dos grupos que se podrían identificar como el actuar de la persona como dueño o directivo de la agencia y el actuar de la agencia como persona jurídica o empresarial. Se espera que el actuar de la persona debe ser con transparencia, decir siempre la verdad y que sea justa. El actuar de la agencia se espera que sea muy solidaria con las otras agencias, ser corresponsable, honesta y actuar con respeto hacia el otro. Ver tabla 2.

Tabla 1. Grado de importancia para integrar un Código de Ética Empresarial

\begin{tabular}{|l|c|}
\hline $\begin{array}{c}\text { Importancia de los principios } \\
\text { hacia una práctica empresarial } \\
\text { con Ética en la AMAV }\end{array}$ & $\begin{array}{c}\text { Evaluación de 1 a 5, } \\
\text { siendo 5 totalmente } \\
\text { de acuerdo. }\end{array}$ \\
\hline Justicia & 4.6 \\
\hline Verdad & 4.7 \\
\hline Respeto & 4.8 \\
\hline Transparencia & 4.7 \\
\hline Corresponsabilidad & 4.5 \\
\hline Honestidad & 4.6 \\
\hline Solidaridad & 4.5 \\
\hline
\end{tabular}

Fuente: Elaborada por los autores (2015)

Importancia de los principios fundamentales para evolucionar hacia una práctica empresarial con ética en la amav.

Con el apoyo del Análisis Discriminante se pudo identificar las variables del grupo que estaría muy interesada en aplicar los principios de la Ética Empresarial en su agencia. Para realizar este análisis se tuvo que bajar la escala de medición de la variable: ¿Cuál es su grado de importancia para integrar un Código de Ética Empresarial en las Agencias de Viajes incorporadas a la AMAV?, medida en escalar intervalar a escala nominal, saliendo dos grupos: 1 los que consideran muy importante y 2 los que consideran importante. Se debe recordar que el cien por ciento contestó entre importante y muy importante. Las variables independientes se fueron seleccionando según su peso relativo y grado de importancia en el modelo discriminante hasta llegar a las siguientes variables que definen mejor el modelo de coeficientes de discriminantes para realizar mejor el pronóstico de los que si estarían muy interesados en implementar la Ética Empresarial:

- Justicia como importante para evolucionar hacia una práctica empresarial ética -0.38

- Respeto con un peso relativo de 0.33

- Honestidad con un peso relativo de 0.33

- Antigüedad en años con un peso relativo de -0.30

- Transparencia con un peso relativo de -0.28

Con una correlación canónica de 0.50, que explica el cien por ciento de la varianza y una lambda de Wilks de 0.75 , que equivale a una chi cuadrada de 52 y con significancia de .000 , se puede concluir que existe suficiente evidencia para definir claramente el comportamiento de dos grupos en función de las variables discriminantes. Es importante resaltar que el $80 \%$ de los casos fueron seleccionados correctamente para realizar este análisis.

Con estos pesos relativos de las variables que miden los principios fundamentales proporcionados por los asociados a la AMAV, se puede calcular la fórmula para predecir la evolución hacia una práctica empresarial con ética en las agencias afiliadas a la AMAV:

$Z=$ Justicia (-.34) + Respeto $(0.34)+$ Honestidad $(0.33)+$ Antigüedad (-0.30) + Transparencia (-0.28)

Tabla 2. Principios fundamentales para la actuación individual e integrupal en el proceso de integrar el código de ética empresarial en la AMAV-México

\begin{tabular}{|l|l|}
\hline \multicolumn{1}{|c|}{ Actuar de la persona o directivos } & \multicolumn{1}{c|}{ Actuar de las agencias de la AMAV } \\
\hline Actuar con TRANSPARENCIA con un peso de .95 & Actuar con SOLIDARIDAD con un peso de .94 \\
\hline Actuar con VERDAD: .90 & Actuar con CORRESPONSABILIDAD: .89 \\
\hline Actuar con JUSTICIA: .55 & Actuar con HONESTIDAD con un peso de .67 \\
\hline & Actuar con RESPETO con un peso de .415 \\
\hline
\end{tabular}

Fuente: Elaborada por los autores (2015) 
Ejemplo: Si partimos de datos hipotéticos de una empresa afiliada a la AMAV-México, con 20 empleados, con evaluación de 5 en justica, respeto, honestidad y transparencia, encontraremos el siguiente valor:

$$
\begin{gathered}
z=5 x(-.337)+5 x(.336)+5 x(.333)+20 x \\
x(-.304)+5 x(-.276)=-5.8
\end{gathered}
$$

Este valor de 5.8 sería el más alto para ubicar a la empresa con alta probabilidad para trabajar con los principios de la Ética Empresarial. Si multiplicamos con 1 cada uno de los valores nos permitiría ubicar a la empresa con la menor disposición a trabajar con ética dentro de la AMAV.

\section{Análisis de prueba de hipótesis hacia la ética empresarial}

\section{Hipótesis I}

- Ho1: No hay diferencia significativa entre las variables propias de las empresas de la AMAV-México y el grado de importancia para integrar el enfoque ético-sostenible en sus negocios.

- Ha1: Si hay diferencia significativa entre las variables propias de las empresas de la AMAV-México y el grado de importancia para integrar el enfoque ético-sostenible en sus negocios.

Si existe diferencia estadística en relación a la forma jurídica con una significancia de .025, por lo que se rechaza la hipótesis nula ya que las empresas constituidas como jurídicas consideran muy importante integrar un código de ética empresarial en su quehacer empresarial; la antigüedad con un grado de significancia de .000, por lo que rechaza la hipótesis nula planteada debido a que todas consideran muy importante integrar un código de ética empresarial; Y el número de empleados con una significancia de .000 señala que las de mayor número de empleados consideran muy importante integrar el Código de Ética Empresarial.

\section{Hipótesis II}

- Ho2: No hay diferencia significativa entre las variables propias de los fundadores de las empresas afiliadas a la AMAV-México y el grado de importancia para integrar el enfoque ético-sostenible en sus negocios.

- Ha2: Si hay diferencia significativa entre las variables propias de los fundadores de las empresas de la AMAV-México y el grado de importancia para integrar el enfoque ético-sostenible en sus negocios.
En cuanto a la relación entre el cargo, se rechaza la hipótesis con una significancia de .000 , ya que los de mayor cargo consideran muy importante integrar el enfoque ético sostenible en sus negocios: en cuanto a la variable género también se rechaza la hipótesis planteada con una significancia de .000 ya que las mujeres están totalmente de acuerdo que se implemente el Código de Ética Empresarial en la AMAV, obviamente secundadas por los del género masculino. Serían las mujeres empresarias de la AMAV-México, las más sensibles para invitarlas a ser parte del equipo de trabajo para diseñar el Código de Ética Empresarial; En cuanto a la edad se puede observar que los jóvenes son los más sensibles para invitarlos a ser parte de los equipos de trabajo con una significancia de .003 y en cuanto al grado de escolaridad no existe gran diferencia ya que sin importar el grado de escolaridad todos consideran de muy importante integrar el Código de Ética Empresarial en su quehacer diario como afiliado de la AMAV.

\section{Hipótesis III}

- Ho3: No hay diferencia significativa entre la actitud de los Presidentes y/o Directivos Generales de las AMAV-México y el grado de importancia para integrar el enfoque ético-sostenible en sus negocios.

- Ha3: Si hay diferencia significativa entre la actitud de los Presidentes y/o Directivos Generales de las AMAV-México y el grado de importancia para integrar el enfoque ético-sostenible en sus negocios.

Se rechaza la hipótesis nula ya que el cien por ciento de los encuestados manifestaron necesitar un Código de Ética Empresarial para la AMAV. Existe la actitud y la voluntad política para integrarlo hacia adentro de la AMAV-México, ya que la evaluación de 4.81, siendo 5 la máxima evaluación nos dice que existe un alto grado de importancia para integrar el código de ética empresarial en las agencias incorporadas a la AMAV. Con una significancia de .000 se acepta la hipótesis alterna ya que los Presidentes y/o Directivos Generales de la AMAV consideran muy importante integra un Código de Ética Empresarial.

Con el análisis discriminante se pudo obtener los factores que miden los principios fundamentales para evolucionar hacia una práctica empresarial con ética en la AMAV y de esta manera precalificar a las agencias para clasificar aquellas que tengan en estos momentos una mayor disposición para trabajar con principios éticos. La fórmula que ayuda a calificar los valores " $Z$ " integra las siguientes variables según su peso relativo: Justicia (-0.38), a mayores práctica empresariales justas se podría observar una actitud más positiva de la Agencia 
para trabajar con un Código de Ética Empresarial; Respeto (0.34), lo que muestra el nivel de exigencia de todo afiliado a tener un comportamiento respetuoso en todos los procesos de negociación; Honestidad (0.33), la evaluación más alta de la variable de honestidad contribuye sin duda alguna a ser una empresa honesta en el trato verbal y escrito en todo el proceso de las negociaciones; antigüedad (-0.30), a mayor antigüedad de la agencia sin duda alguna contribuye con los buenos deseos de los afiliados ya que es necesario que se compartan los puntos fuertes o fortalezas entre agencias para otorgar un servicio en equipo de calidad y por último la transparencia (-0.28), obviamente a mayor transparencia en el actuar diario de la agencia facilitará el proceso de integración de proyectos para atender a mercados y clientes comunes: $Z=$ Justicia $(-0.34)+$ Respeto $(0.34)+$ Honestidad $(0.33)+$ Antigüedad $(-0.30)+$ Transparencia $(-0.28)$

\section{Conclusiones}

Los resultados arrojados por la investigación de fuente primaria con el instrumento que se aplicó permitió construir un marco de referencia para integrar el código de ética sostenible que contribuya a la implementación de la Responsabilidad Social empresarial integrada en la ISO 26000 para las Agencias de Viajes afiliadas a la AMAV-México.

Con los resultados se logra el objetivo principal de este artículo que es el de establecer consideraciones el código de ética empresarial, con base en el grado de importancia que tiene para las empresas afiliadas a la AMAV-México, integrar el enfoque ético sostenible, para proponer unas consideraciones para el código de ética empresarial, para integrar el enfoque ético sostenible en las empresas afiliadas a la AMAV-México.

Los factores internos de cada Agencia de Viajes para tener en cuenta las consideraciones para el código de ética empresarial son: actuar con profesionalismo, actuar con la verdad, actuar de manera responsable, todas las agencias deben mejorar el servicio e involucrar a todo el personal que labora en la Agencia, así como integrar los elementos éticos en la Misión, Visión y políticas de la empresa. Los elementos concretos sugeridos por los afiliados de la AMAV a integrar en el Código de Ética son en orden de importancia: honestidad, respeto, transparencia, calidad del servicio, amistad, comunicación, confianza, solidaridad y responsabilidad.

Los afiliados a la AMAV, si ven la necesidad de integrar un Código de Ética Empresarial y lo consideran importante para actuar éticamente ante los socios mismos, ante sus clientes, proveedores, ante sus empleados y por último ante el Presidente de la AMAV, lo que se puede interpretar como un deseo de actuar por beneficio propio y beneficio de grupo y no para quedar bien ante las autoridades de la misma Asociación.

Los Factores para la negociación que se deben tomar en cuenta para transacciones entre las agencias de viajes son por orden de importancia: el respeto, saber trabajar en equipo, buscar siempre satisfacer las necesidades turísticas de los clientes, actuar siempre con honestidad, transparencia, solidaridad y la comunicación oportuna y suficiente para tomar decisiones en beneficio de los clientes y de las agencias de viajes.

\section{Consideraciones para un código de ética empresarial}

Para que sirva como consideraciones para el Código de ética empresarial para las empresas afiliadas a la AMAV-México, que inicien los pasos para integrarlo con el sello distintivo de la Asociación AMAV-México, se propone, teniendo en cuenta a Debeljuh (2005) que el verdadero cometido de un código de ética ha de ser ayudar a la excelencia en el trabajo de todas las personas y, en concreto, facilitarles el desarrollo de sus virtudes y el despliegue de todas sus potencialidades:

\section{Consideraciones para el código de ética que refleje la conducta moral sostenible de los afiliados en función de:}

- Mostrar con palabras sencillas lo ético de AMAV

- Principios y/o valores definidos por los afiliados de la AMAV

- Normas y reglas para implementar el código de ética:

- Regular y dirigir el comportamiento de todos

- Definir obligaciones

- Premios y castigos

- Responsabilidad con los resultados

También es necesario tener claridad que siendo el Código Ético un instrumento que regula el comportamiento de la totalidad de las personas naturales o jurídicas de una organización, él debe adquirir un carácter de herramienta estratégica de acción y, como todo lo estratégico, solo puede ser redactado y aprobado por personas que ocupen ese nivel y tengan el poder moral y legal suficiente para hacerlo cumplir. 
Sostenibilidad con la implementación

de la responsabilidad social empresarial con acciones concretas en:

- Crecimiento económico responsable

- Práctica de la mercadotecnia ética

- Precio justo

- Publicidad no engañosa

- Producto orientado a las necesidades del cliente, etc.

- Contribución al medioambiente

- Programas para preservar los espacios del turismo extremo como bosques, ríos, etc.

- Programas de reciclaje permanente

- Contribución social

- Programas para disminuir la pobreza

- Salarios dignos

Para fines prácticos se ha diseñado el siguiente esquema gráfico para una mejor comprensión del modelo ético sostenible para la AMAV. Que dando en el centro del modelo los elementos esenciales para la ética empresarial como la formulación de los valores y principios fundamentales para integrar la ética en el quehacer diario de todos los empleados de las agencias afiliadas a la AMAV, formulación de políticas que permitan desarrollar e implementar el contenido del código de ética, definir los lineamientos de la responsabilidad con los resultados obtenidos en el diario vivir del trabajo y especificar las normas y reglas del juego para implementar el código. Por la parte exterior se definen los pilares fundamentales de la RSE, como es el trabajar por un desarrollo económico responsable, protección y cuidado del medio ambiente, contribución social, resultados e impacto interno y externo. Ver figura 8.

Con el apoyo de este esquema de la ética sostenible, se va a ejemplificar con los resultados obtenidos en uno de los elementos de la ética y de la RSE para las Agencias de Viajes afiliadas a la AMAV-México:

\section{Valores y principios:}

a) Actuar de la persona o de la agencia: con transparencia, verdad, justicia y solidaridad.

b) Actuar entre agencias de viajes y o grupos de la AMAV: con solidaridad, corresponsabilidad, honestidad y con respeto.

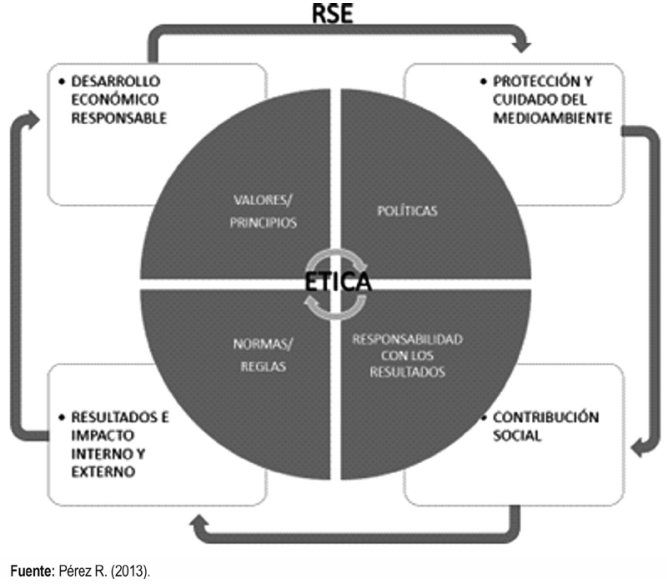

Figura 8. Ética sostenible empresarial para la AMAV-México

2. Políticas: Actuar con la verdad, respeto, justicia, transparencia, honestidad, solidaridad y corresponsabilidad de todos los actos comerciales y no comerciales entre los afiliados y para con todos los grupos de interés de la AMAV.

3. Responsabilidad con los resultados: ser corresponsable en la proporción de la interacción comercial o transaccional entre agencias de viajes, en beneficio siempre del cliente que se comparta durante todo el proceso del servicio turístico.

\section{Normas y Reglas:}

a) Para los empleados y todas las personas involucradas en los procesos de la prestación del servicio de la Agencia de Viaje vinculadas a la AMAV.

b) Ante la gestión medio ambiental

c) Ante el impacto social

d) Ante el trabajo en equipo que se debe desarrollar a nivel nacional entre todos los afiliados a la AMAV, para tener un frente común en la atención de los clientes.

Cada uno de estos cuatro puntos clave para el desarrollo de la ética empresarial debe ser tratado con la participación directa de todos los afiliados bien sea con el apoyo de convenciones o bien de manera asincrónica con el apoyo de la tecnología, pero que en verdad se incluyan todas las ideas y pensamientos alrededor de la ética, ya que todo documento ético de éxito depende en gran medida de la integración de todas las ideas y pensamientos de todos los empleados de una organización para generar sentido de pertinencia y empo- 
deramiento de un documento que no vaya a quedar en letra muerta plasmada en algún tipo de decálogo o pasaporte ético empresarial.

5. En la parte exterior del esquema se puede apreciar en primera instancia y siguiendo el esquema como las manecillas del reloj, el desarrollo Económico Responsable:

a) Integrar las ideas u opiniones de todos los accionistas de la empresa, ya que es el capital de ellos el que está en juego en el desarrollo de la empresa.

b) Ver las actividades de RSE como una oportunidad para desarrollar nuevos productos $y$ mercados muy rentables y por ende generará mejores beneficios económicos para la empresa. Para los directivos de las Agencias de Viajes de la AMAV evaluaron con 4.2, siendo 5 totalmente de acuerdo el que la RSE permite generar beneficios económicos.

c) Actuar de manera responsable en todo momento de crecimiento y desarrollo económico de la agencia y de sus proveedores, así como buscar siempre el beneficio de sus clientes.

d) Desarrollar actividades para impulsar el comercio justo

e) Fomentar un consume responsable.

6. Protección y cuidado del medioambiente: los mismos afiliados a la AMAV sugieren realizar acciones de elaboración y uso de productos verdes, establecer programas de reciclaje, compromiso con el uso de energía limpia y renovable.

7. Contribución social: fomentar políticas de no discriminación laboral, impulsar planes de desarrollo para los empleados y protocolos para integración de mano de obra extranjera.

\section{Recomendaciones}

Para empezar a realizar actividades éticas en la AMAVMéxico, se recomienda un actuar deseado para cada uno de los miembros socios y/o afiliados que cumplan con los principios de transparencia en todo el proceso de la transacción comercial con clientes, proveedores y entre agencias afiliadas o no a la AMAV, decir siempre la verdad y actuar con justicia apelando al buen juicio personal de lo que es justo o no en todo el proceso de las transacciones comerciales procurando el ganar-ganar.
Se sugieren los siguientes principios fundamentales para actuar como equipo de trabajo AMAV: actuar con solidaridad, esto es, existen agencias con mayores fortalezas y ventajas competitivas que deberían colaborar con las agencias más pequeñas o medianas, ser corresponsables con todos los socios involucrados en proyectos turísticos para deslindar la responsabilidad en proporción de la participación de cada una de las agencias involucradas en los proyectos turísticos. Actuar con honestidad y respeto para construir la confianza que hoy se necesita para poder atender de manera transparente a nuestros clientes, proveedores y grupos de interés involucrados en todas las agencias afiliadas a la AMAV.

Dentro de las acciones inmediatas y posibles a realizar para los afiliados de la AMAV-México, están: establecer un código de conducta escrito en la empresa, desarrollo de planes de formación para los empleados de la Agencia de Viajes, desarrollo de programas para la prevención y evaluación de riesgos laborales, información a los clientes para fomentar un consumo responsable, desarrollo de actividades para impulsar el comercio justo, protocolos que garanticen la veracidad de la publicidad de la empresa, fomento de políticas no discriminatorias en la contratación de trabajadores y establecer programas de reciclaje.

La eficacia de un código de ética para Debeljuh (2005) se relaciona estrechamente con el grado de compromiso de las personas con los valores que él contiene. En la medida en que cada persona se decida libre y conscientemente a ser leal y coherente con esas pautas de acción y oriente su conducta hacia ellas, ese código será eficaz. Sólo de esta manera se podrá mostrar que, a través de ese documento, se ha logrado educar a la persona en el desarrollo de sus virtudes.

De esta manera para Debeljuh (2005) el verdadero cometido de un código de ética ha de ser ayudar a la excelencia en el trabajo de todas las personas y, en concreto, facilitarles el desarrollo de sus virtudes y el despliegue de todas sus potencialidades. Sólo así, todo código, lejos de consistir en un mero andamiaje externo, se apoya e incide en el interior de cada sujeto y acaba convirtiéndose en un verdadero modo de ser enraizado en la virtud. Así como la ética se hace vida en las virtudes de las personas, del mismo modo, un código de conducta debe traducirse en el talante moral de todos los que se relacionan con él. Mediante este documento, la ética - que es una e indivisible como es la verdad sobre el hombre- se concreta en el ejercicio libre y responsable del obrar humano dentro de las organizaciones, abriendo cauces para el creci- 
miento en las virtudes. Si el código de ética logra este objetivo, habrá hecho frente a su único desafío.

Finalmente, los afiliados de la AMAV-México, requieren de unas consideraciones para el Código de ética empresarial para implementarlo en el marco de la RSE con el sello distintivo de la AMAV-México o de ser posible se debe trabajar de manera urgente el concepto de la sostenibilidad debido a que este integra la ética empresarial y las actividades de Responsabilidad Social Empresarial.

\section{Referencias}

Álvarez Rivas D, De La Torre Diaz J. (2002): Empresas des-almadas? Una Visión Ética del Mundo Empresarial, Dykinson.

Argandoña A. (1990). "Necesidad y 'Rentabilidad' de la ética en los negocios, Cultura y Ética Empresariales". Círculo de Empresarios. Boletín 50 número 2.

Bastidas O. (2014) Bases para la formulación del código de ética de Fedecámaras. Plan de acción. Comisión de responsabilidad social de Fedecámaras. Venezuela.

Cano J. (2011) Percepción de la ética y la responsabilidad social empresarial en los ejecutivos de Monterrey, tesis grado de maestría en ciencias, Universidad Autónoma de Nuevo León Facultad de Filosofía y Letras, Monterrey México.

Cortina A. (coord.) (1996): Ética de la organización. Claves para una nueva cultura empresarial, Madrid, Trotta (2.a edición).

De George R. (2011) Historia de la ética empresarial,https://www. bbvaopenmind.com/articulo/historia-de-la-etica-empresarial/? fullscreen=true, consultado el 9 de diciembre de 2015.

Debeljuh P. Los códigos de ética en las empresas en E \& G Economía e Gestão, Belo Horizonte. 2005;5(9):48-56.

Deck M. (2002) "Corporate Codes and Ethics Programs", en Hartman, L.P., Perspectives in Business Ethics, McGraw-Hill Irwin, New York.

Echaniz T. (2003) Ideas para la formación en ética empresarial, Lan Harremanak/9 (2003-II) (51-59)

Falçao H, Fontes J. (1999). ¿en quién se pone el foco? Identificando stakeholders para la formulación de la misión organizacional, Revista Reforma y Democracia № 15 octubre. [Acceso: 10 de diciembre de 2015] http://www.bdp.org.ar/facultad/catedras/ cp/analisispp/Falcao\%20y\%20Fontes\% 20Filho.pdf

Fisher K, Geenen J, Jurcevic M, McClintock K, Davis G. Applying asset-based community development as a strategy for CSR: a Canadian perspective on a win-win for stakeholders and SMEs. Business Ethics: A European Review. 2009;18(1):66-82.

Freeman R, Reed D. Stockholders and Stakeholders: A New Perspective on Corporate Governance-California Management Review, Spring. 1983;25(3):88- 106.

Freeman R. (1984) Strategic Management: A Stakeholder Analysis. Boston: Pittman.
Gaumnitz B, Lere J. "A Classification Scheme for Codes of Business Ethics". En: Journal of Business Ethics. 2004;49:329-335.

Giddens A. (1979). Central Problems in Social Theory: Action, Structure and Contradiction in Social Analysis. California: Berkley University.

Gil A, Luciano P. La gestión de los grupos de interés: una reflexión sobre los desafíos a los que se enfrentan las organizaciones en la búsqueda de la sostenibilidad empresarial, Revista de métodos cuantitativos para la economía y la empresa 2011;71-90.

Gómez Pérez R. (1990). "Ética Empresarial: Teoría y Casos". Ed. Rialp, Madrid.

González E, García D. La Responsabilidad Soda1 Empresarial (RSE) en Europa: la apuesta por un nuevo modelo de empresa. Una revisión crítica desde la ética empresarial, Recerca Revista De Pessament I ANALISIS. 2006:157-170.

Graafland J, Ven van de B, Stoffele N. Strategies and Instruments for Organising CSR by Small and Large Businesses in the Netherlands. Journal of Business Ethics. 2003:47(1):45-60.

Guillen M. La ética empresarial: una aproximación al fenómeno, en cuadernos empresa y humanismo. 2010;58:1-39.

Herrera B. (2010). Responsabilidad social empresarial y acceso de las empresas a la economía global. En C. Martínez, Responsabilidad social empresarial: una reflexión desde la gestión, lo social y ambiental (págs. 54-55). Bogotá: Centro Editorial, FCE - Universidad Nacional de Colombia

Herrera J, Abreu J. Cómo gestionar la responsabilidad social en las pymes colombianas, Daena: International Journal of Good Conscience. 2008;3(1):395-425.

Larrán M, Herrera J, Lechuga MP. 2011. El Estado del arte en la investigación sobre Responsabilidad Social y PYMES. In: XVI Congreso AECA Nuevos modelos económicos: Empresa, Mercados y Culturas. Granada, España 21-23 September.

Lozano JF. "Proposal for a model for the elaboration of ethical codes based on discourse ethics", Business Ethics: A European Review. 2001;10(2):157-162.

MacDonald C. (2010). The business ethics blog: Ethics definition. The Business Ethics Blog, [blog] 21 March. Available at: http:// businessethicsblog.com/2010/03/21/ethics-definition/ [Acceso diciembre 10 de 2015].

Martin L. Acuerdos y normas. Los códigos en la ética empresarial, Telos. 2000;2(2):278-283.

Melé D. (1991). "Ética y Empresa". Información Comercial Española, marzo, n691. MENGUZZATO

Mohn R. (2005) La responsabilidad social del empresario. Galaxia Gutenberg, Círculo de Lectores. Barcelona, p. 90.

Montuschi L. (2010) Los códigos de ética como instrumentos de la responsabilidad social corporativa, RPDE

Nascimento O. (2000) Os códigos de ética empresariais - instrumentos motivadores de comportamentos éticos, Encontros científicos Universidade do Algarve, Portugal. 
Nielsen AE, Thomsen C. Investigating CSR communication in SMEs: a case study among Danish middle managers. Business Ethics: A European Review. 2009;18(1):83-93

Pérez L. (2013) Las Pymes ante el reto de la sostenibilidad, Revista Líder Empresarial, México, 2013; http://www.liderempresarial. com/hojeable.p hp?num=216

Perrini F. SMEs and CSR Theory: Evidence and Implications from an Italian Perspective. Journal of Business Ethics. 2006;67(3):305316.

Post J, Preston L, Sachs S. Managing the extended enterprise: the new stakeholder view, California Management Review. 2002;45(1):6 28.

Post J, Preston L, Sachs S. (2002b). Redefining the corporation. Stakeholder management and organizational wealth, California: Stanford University Press.

Ramos K. (2013) La responsabilidad social empresarial de las entidades del sector salud: descripción y análisis crítico de la RSE en los códigos de buen gobierno, ética y conducta de las principales entidades promotoras de salud colombianas, Trabajo de grado de la Maestría en Administración, Universidad Nacional de Colombia Facultad de Ciencias Económicas, Bogotá, Colombia.

Román B. (2000) La dimensión ética de la práctica empresarial. En ARS BRWIS, Pp. 209-227.
Russo A, Tencati A. Formal vs. Informal CSR Strategies: Evidence from Italian Micro, Small, Medium-sized, and Large Firms. Journal of Business Ethics. 2009;85 (2 Supplement):339-353.

Schwartz M. "Universal moral values for corporate codes of ethics". En: Journal of Business Ethics. 2005;59:27-44.

Spence LJ, Lozano JF. Communicating about Ethics with Small Firms: Experiences from the U.K. and Spain. Journal of Business Ethics. 2000;27(1):43-53.

Spence LJ, Rutherfoord R. Small Business and Empirical Perspectives in Business Ethics: Editorial. Journal of Business Ethics. 2003;47(1):1-5.

Suarez L. (2011) Ética Empresarial y RSE: Tendencias desde Chile, Razón Práctica y Asuntos Públicos. Revista de Ética y Filosofía Política

Vázquez-Carrasco R, López-Pérez ME. 2012. Small \& medium-sized enterprises and Corporate Social Responsibility: a systematic review of the literature. Quality and Quantity, [online] Available at: http://www.springerlink.com/content/6331703p64647561/ [Acceso 10 de diciembre 2015].

Worthington I, Ram M, Jones T. 'Giving something back': a study of corporate social responsibility in UK South Asian small enterprises. Business Ethics. 2006;15(1):95-108. 